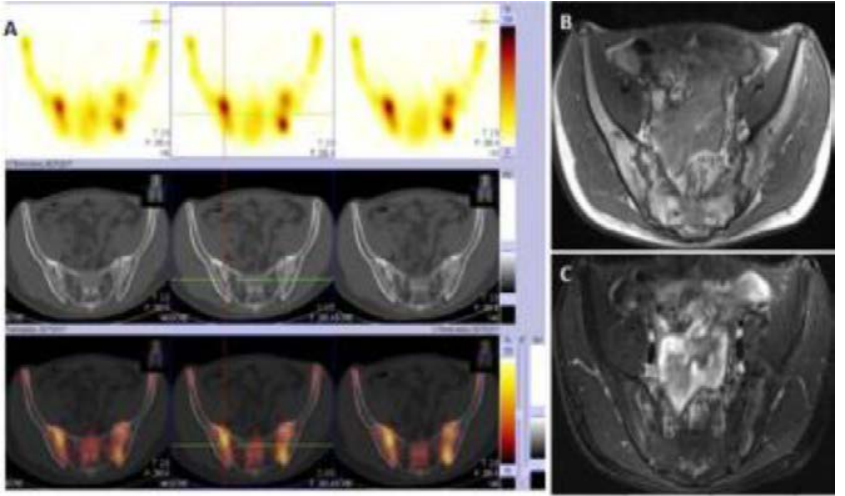

Figure 1. An 20-years-old man with 5 years of low back pain and spine malformation. (A) SPECT-CT showed an abnormal concentration of radioactivity in SIJ. (B-C) In SIJ, MRI showed a high signal on T1-WI, and a high signal on STIR.
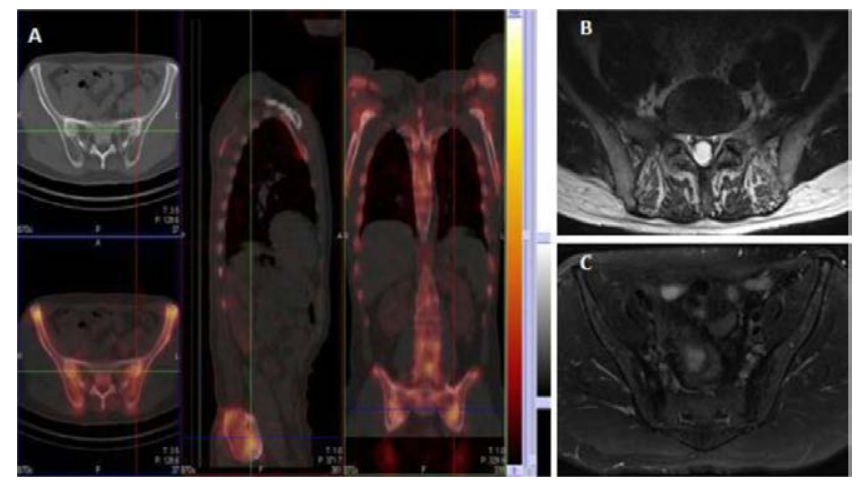

Figure 2. An 37-year-old man with 20 years of low back pain and spine malformation. (A) SPECT-CT showed an abnormal concentration of radioactivity in SIJ. (B-C) In SIJ, MRI showed a high signal on T1-WI, and a low signal on STIR.

Disclosure of Interests: Qing Han: None declared, Zhaohui Zheng: None declared, Kui Zhang: None declared, Zheng Yu: None declared, Fengfan Yang: None declared, Qiang Liang: None declared, Ping Zhu: None declared, Xenofon Baraliakos Grant/research support from: Grant/research support from: AbbVie, BMS, Celgene, Chugai, Merck, Novartis, Pfizer, UCB and Werfen, Consultant of: AbbVie, BMS, Celgene, Chugai, Merck, Novartis, Pfizer, UCB and Werfen, Speakers bureau: AbbVie, BMS, Celgene, Chugai, Merck, Novartis, Pfizer, UCB and Werfen

DOI: 10.1136/annrheumdis-2020-eular.3154

\section{SAT0564 BONE TEXTURE ANALYSIS WITH DEEP LEARNING IN HAND RADIOGRAPHS FOR ASSESSING THE RISK OF RHEUMATOID ARTHRITIS}

C. F. Kuo ${ }^{1,2}$, S. Miao ${ }^{3}$, K. Zheng ${ }^{3}$, L. Lu ${ }^{3}$, C. I. Hsieh ${ }^{1}$, C. Lin ${ }^{2} .{ }^{1}$ Chang Gung Memorial Hospital, Division of Rheumatology, Allergy and Immunology, Taoyuan, Taiwan, Republic of China; ${ }^{2}$ Chang Gung Memorial Hospital, Center for Artificial Intelligence in Medicine, Taoyuan, Taiwan, Republic of China; ${ }^{3}$ PAll labs, Bethesda, United States of America

Background: Conventional $x$-rays are essential to identify radiographic changes of rheumatoid arthritis (RA) in structure and bone texture. Limited evidence suggests that the bone texture analysis may quantify the radiographic changes in $\mathrm{RA} ;{ }^{1}$ however, current techniques such as the fractal dimension characterize fixed texture features. Deep learning offers novel methods to 'learn' radiographic texture features relevant to RA.

Objectives: To develop a deep learning model to assess the radiographic bone texture in the distal metacarpal bone relevant to RA.

Methods: We collected 3,738 conventional hand radiographs from 2,128 individuals (RA, $n=908$; non-RA, $n=1220$ ). The second, third, and fourth metacarpal bone images were segmented using a curve Graph Convolutional Network (GCN), and the distal third was used as the input to train a texture model to classify RA. The texture model was based on the Deep Texture Encoding Network (Deep-TEN) architecture (figure 1), ${ }^{2}$ which put an encoding layer on top of a pre-trained 18-layered residual network (ResNet18). The vectors produced by the model represent the orderless texture features that were used to generate a texture score for RA.
Five texture models are trained using 5 -fold cross-validation and are ensembled during inference by averaging the model outputs to produce the final score. We then validate the model using hand radiographs of 166 RA patients and 166 non-RA patients. Overall model performance was measured by area under the curve of the receiver operator curve (AUROC). Multivariate logistic regression was used to estimate the odds ratio (OR) and $95 \%$ confidence interval $(\mathrm{Cl})$ of RA.
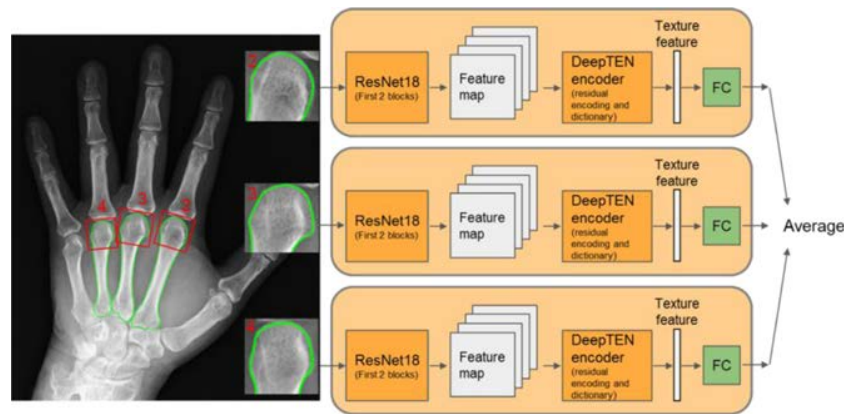

Figure 1. Schematic representation of deep learning models to extract and encode texture features for RA classification.

Results: We included 140 women and 26 men with RA (mean age, $55.9 \pm 1.8$ years) and 166 non-RA individuals ( $F: M, 140: 26$; mean age, $55.5 \pm 1.8$ years). The mean texture score was $0.49(95 \% \mathrm{Cl}, 0.48-0.50)$ in RA patients, which is significantly higher than non-RA patients $(0.42,95 \% \mathrm{Cl}, 0.40-0.43 ; \mathrm{p}<0.01)$. The AUROC of the model was 0.68 . In the multivariate logistic regression model, a high texture score $(>0.43)$ is associated with an OR $(95 \% \mathrm{Cl})$ of $3.42(2.48-4.72)$ for RA, adjusted by age and sex.

Conclusion: This study indicates that the texture model can delineate radiographic changes in texture relevant to RA and, coupled with automatic joint detection and segmentation, it has the potential to aid early RA diagnosis and monitor radiographic progression.

References:

[1] Zandieh S, Haller J, Bernt R, et al. Fractal analysis of subchondral bone changes of the hand in rheumatoid arthritis. Medicine (Baltimore) 2017;96(11):e6344.

[2] Zhang H, Xue J, Dana K. Deep TEN: Texture Encoding Network. The IEEE Conference on Computer Vision and Pattern Recognition (CVPR) 2017:708-17. Disclosure of Interests: None declared

DOI: 10.1136/annrheumdis-2020-eular.837

\section{SAT0565 MODIFIED ULTRASONOGRAPHY SCORING SYSTEM BASED ON ORAL MUCOSA AND MAJOR SALIVARY GLANDS IMPROVING THE DIAGNOSTIC SPECIFICITY OF PRIMARY SJÖGREN'S SYNDROME}

Y. Liu' ${ }^{1}$, S. Zhang ${ }^{1}$, W. Li ${ }^{1}$, J. Zhu ${ }^{1} .^{1}$ Peking University People's Hospital, Department of Ultrasound, Beijing, China

Background: Primary Sjögren's syndrome (pSS) is a chronic autoimmune disease that affects exocrine systems. Xerostomia is one of representative clinical features which contributes to an accelerated destruction of the mucosal epithelium. Atrophic lesions of the oral mucosa is a common observation in pSS cases. Objectives: To develop a modified ultrasonography scoring system which included the buccal mucosa and major salivary glands in patients with pSS and assess the ultrasonography characteristics of buccal mucosa.

Methods: Ultrasonography of buccal mucosa and major salivary glands were conducted for $53 \mathrm{pSS}$ patients and 44 patients with SS-related manifestation but without pSS. The thickness of buccal mucosa and the echogenicity of the buccal mucosa were scored using the 0-2 scoring system. The sonographic features of major salivary glands were scored using the $0-16$ scoring system. Differences of buccal mucosa thickness between groups were compared with Mann-Whitney test. The 2 methods (0-16 scoring system, modified 0-18 scoring system) were compared and statistical cutoffs were identified using receiver operating characteristic (ROC) curve analysis.

Results: The thickness

$r$ the pSS group was significantly thinner than that for the non-pSS group $(p<$ 0.001 ), and the best score cut-off was $0.38 \mathrm{~mm}$. The echogenicity of buccal epithelium was heterogeneously hyperechoic in $32(60.4 \%)$ of the 53 patients, and was $5(11.4 \%)$ of the control group. The optimal cutoff point to distinguish between pSS and non-pSS was 7 in the modified scoring system (sensitivity $79.2 \%$, specificity $95.5 \%$ ), and it was 6 in the $0-16$ system (sensitivity $81.1 \%$, specificity $86.4 \%$ ). Compared with the $0-16$ system, modified scoring system improved the diagnostic specificity.

Conclusion: The thickness of the oral mucosa in patients with pSS is thinner and the echogenicity in buccal epithelium increases compared with that of non-pSS. 
The modified ultrasonography scoring system integrated oral mucosa and major salivary glands is able to improve the diagnostic specificity of patients with pSS.

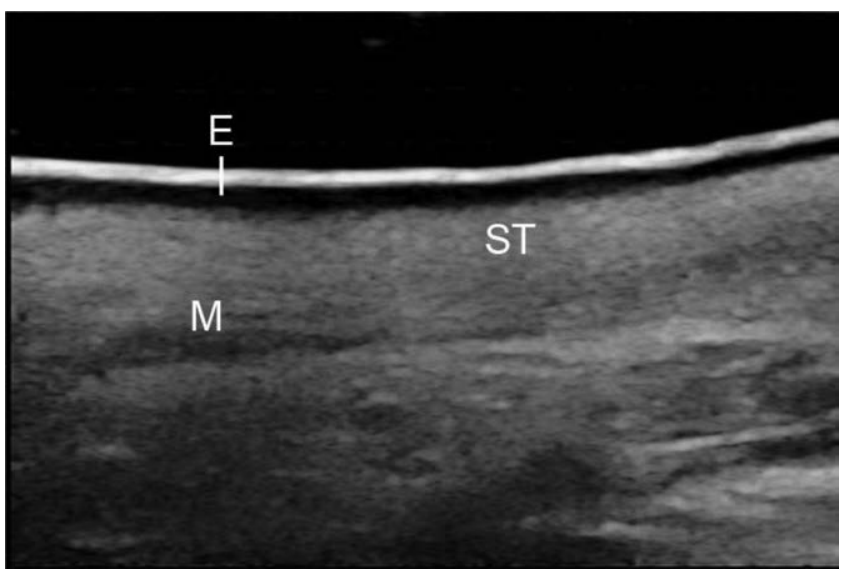

Figure 1. Ultrasonography image of the normal buccal tissue. $E$ = epithelial membrane of buccal mucosa; ST = subepithelial connective tissue; $\mathrm{M}$ = buccinator muscle.

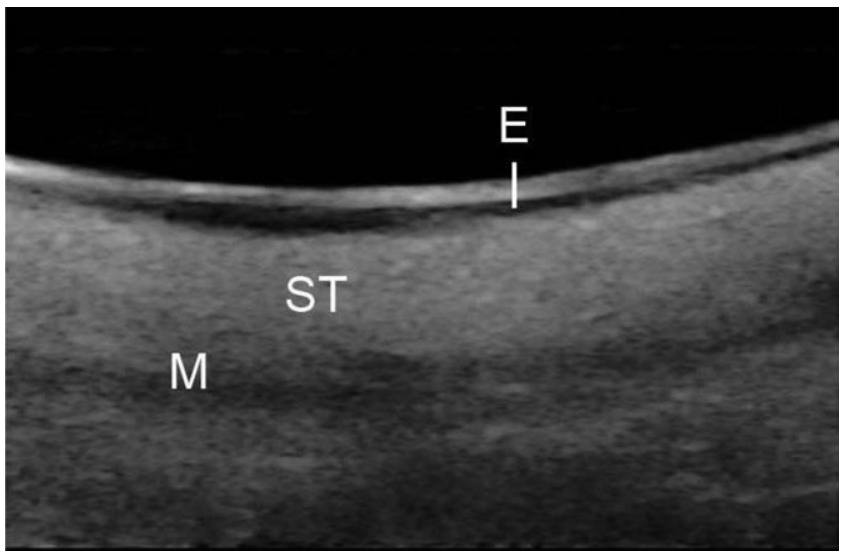

Figure 2. Ultrasonography image of pSS patient reveals decreased thickness and heterogeneous hyperechogenicity of buccal mucosa. $\mathrm{E}=$ epithelial membrane of buccal mucosa; $\mathrm{ST}=$ subepithelial connective tissue; $\mathrm{M}$ = buccinator muscle.

References:

[1] Belkacem Chebil R, Oueslati Y, Marzouk M, Ben Fredj F, Oualha L, Douki N. Oral Lichen Planus and Lichenoid Lesions in Sjögren's Syndrome Patients: A Prospective Study. Int J Dent. 2019:1603657.

[2] De Vita S, Lorenzon G, Rossi G, Sabella M, Fossaluzza V. Salivary gland echography in primary and secondary Sjögren's syndrome. Clin Exp Rheumatol 1992;10(4):351-6.

[3] Liu Y, Zhu J, Guo H, Li W, Li J, Wang Y, et al. Buccal Mucosa Assessed by Ultrasonography in Healthy Adults: Methodology and Determination of Normal Appearance. Ultrasound Med Biol. 2019;45(5):1297-1305.

Acknowledgments: This work was partly supported by National Natural Science Foundation of China (No. 81571684)

Disclosure of Interests: None declared

DOI: 10.1136/annrheumdis-2020-eular.4012

\section{SAT0566 ULTRASOUND DOPPLER MASEI SHOWS SENSITIVITY TO CHANGE AFTER BIOLOGICAL THERAPY IN SPONDYLOARTHRITIS AND PSORIATIC ARTHRITIS PATIENTS}

J. Molina Collada ${ }^{1}$, C. Macía-Villa ${ }^{2}$, C. Plasencia ${ }^{3}$, J. M. Alvaro-Gracia ${ }^{1}$, E. De Miguel ${ }^{3} .{ }^{1}$ Hospital Universitario Gregorio Marañón, Madrid, Spain; ${ }^{2}$ Hospital Universitario Severo Ochoa, Madrid, Spain; ${ }^{3}$ Hospital Universitario La Paz, Madrid, Spain

Background: The assessment of activity in spondyloarthritis ( $\mathrm{SpA}$ ) and psoriatic arthritis (PsA) involves several domains, including enthesitis. Clinical enthesitis has shown low sensitivity, specificity and reliability. The MAdrid Sonographic Enthesitis Index (MASEI) is a feasible and reliable ultrasound score, but its responsiveness to treatment has not yet been evaluated.

Objectives: The main objective of this study was to investigate the sensitivity to change of MASEI in active SpA and PsA patients.
Methods: Longitudinal study in patients with SpA and PsA with active disease (defined as patients who were going to start or switch biologic disease modifying antirheumatic drugs (bDMARD) therapy according to physician criteria and in agreement with clinical guidelines). MASEI evaluation was performed at baseline, 3- and 6-months visits. MASEI and Outcome Measures in Rheumatology (OMERACT) enthesitis Power Doppler (PD) definitions were checked. Each enthesis was scanned in both the longitudinal and transverse planes, and $5 \mathrm{sec}$ ond videos were recorded for reliability. An inter-reader analysis by three readers was performed. For statistical analysis t-Student test was used to determine changes between visits and kappa test was used for reliability.

Results: A total of 72 US evaluations of 25 patients were included, of whom $13(52 \%)$ were ankylosing spondylitis (AS) patients, 9(36\%) PsA, and 3(12\%) non radiographic axial spondyloarthritis (nr-axSpA). Mean age was $51.2 \pm 14.1$ years and $13(52 \%)$ were females. Mean DAS28 $(3.5 \pm 1.2)$ for peripheral involvement, mean BASDAI (5.8 \pm 2$)$ for axial involvement, and CRP values $(13.1 \pm 13.6)$ reflect moderate-high disease activity at baseline. US parameters at baseline and at the 3- and 6-month follow-up visits are shown in Table 1. Global MASEI score was responsive at the 3- and 6-month follow-up visit $(-4.9$ and -5.7 , respectively) $(p<0.05)$ and both MASEI and OMERACT PDUS definitions of active enthesitis improved significantly at 3- $(-0.6$ and -1.1$)$ and 6-month follow-up visits $(-0.7$ and -1.1$)(p<0.05)$. Reliability of PD MASEI definition among the three readers was excellent (kappa $=0.918)$.

Table 1. MASEI evaluation at baseline, 3- and 6-month follow-up visits

\begin{tabular}{|c|c|c|c|c|c|}
\hline Parameter & $\begin{array}{c}\text { Baseline } \\
n=25\end{array}$ & $\begin{array}{c}3 \\
\text { months } \\
n=25\end{array}$ & $\mathbf{P}^{\mathrm{a}}$ & $\begin{array}{c}6 \\
\text { months } \\
n=22\end{array}$ & $P^{a}$ \\
\hline MASEI score & $28 \pm 9.3$ & $23.2 \pm 7.6$ & 0.002 & $24.7 \pm 8.1$ & 0.01 \\
\hline PD US MASEI score & $1.8 \pm 1.3$ & $1.1 \pm 1.1$ & 0.046 & $1 \pm 0.9$ & 0.004 \\
\hline PD US OMERACT score & $1.6 \pm 1.2$ & $0.9 \pm 0.9$ & 0.024 & $0.8 \pm 0.9$ & 0.006 \\
\hline
\end{tabular}

$\mathrm{a}_{\mathrm{t}-\text { Student test for comparison to baseline }}$

Conclusion: MASEI score significantly improves at 3 and 6 months of follow up in patients under bDMARD treatment and both MASEI and OMERACT Doppler definitions of active enthesitis reflects treatment response. These findings sup port the usefulness of PD US in the assessment of bDMARD treatment response in SpA and PsA.

Disclosure of Interests: Juan Molina Collada: None declared, Cristina MacíaVilla: None declared, Chamaida Plasencia: None declared, Jose-Maria Alvaro-Gracia Grant/research support from: Abbvie, Elli-Lilly, MSD, Novartis, Pfizer, Consultant of: Abbvie, BMS, Janssen-Cilag, Elli-Lilly, MSD, Novartis, Pfizer Sanofi, Tigenix, Roche, UCB, Paid instructor for: Elli-Lilly, Pfizer, Roche, Speakers bureau: Abbvie, BMS, Janssen-Cilag, Elli-Lilly, Gedeon Richter, MSD, Novartis, Pfizer, Sanofi, Tigenix, Roche, UCB, Eugenio de Miguel Grant/research support from: Yes (Abbvie, Novartis, Pfizer), Consultant of: Yes (Abbvie, Novartis, Pfizer), Paid instructor for: yes (AbbVie, Novartis, Pfizer, MSD, BMS, UCB Roche, Grunental, Janssen, Sanofi), Speakers bureau: yes (AbbVie, Novartis, Pfizer, MSD, BMS, UCB, Roche, Grunental, Janssen, Sanofi) DOI: 10.1136/annrheumdis-2020-eular.6425

\section{SAT0567 USE OF THERMOGRAPHY OF HANDS AND MACHINE LEARNING TO DIFFERENTIATE PATIENTS WITH ARTHRITIS FROM HEALTHY SUBJECTS}

I. Morales-Ivorra ${ }^{1,2}$, D. Grados Canovas ${ }^{1}$, C. Gómez Vaquero ${ }^{3}$, J. M. Nolla ${ }^{3}$, J. Narváez ${ }^{3}$, C. Moragues Pastor ${ }^{3}$, J. A. Narvaez ${ }^{3}$, J. Hernandez ${ }^{3}$, J.

C. Sardiñas ${ }^{3}$, B. Busque ${ }^{1}$, D. Madrid ${ }^{1}$, J. Bové ${ }^{1}$, M. A. Marin-López ${ }^{2}{ }^{1}{ }^{1}$ Hospital Igualada, Igualada, Spain; ${ }^{2}$ Singularity Biomed S. L., Barcelona, Spain; ${ }^{3}$ Hospital Universitari de Bellvitge, Hospitalet de Llobregat, Spain

Background: The early diagnosis of rheumatic diseases improves their prognosis. However, patients take several months to reach the rheumatologist from the beginning of the first symptoms. Thermography is a safe and fast technique that captures the heat of an object through infrared photography. The inflammation of the joints causes an increase in temperature and, therefore, can be measured by thermography. Machine learning methods have shown that they are capable of analyzing medical images with an accuracy similar or superior to that of a healthcare professional.

Objectives: Develop an algorithm that, based on thermographic images of hands and machine learning, differentiates healthy subjects from patients with rheumatoid arthritis (RA), psoriatic arthritis (PA), undifferentiated arthritis (UA) and arthritis of hands secondary to other diseases (SA).

Methods: Multicenter observational study conducted in the rheumatology and radiology service of two hospitals. Patients with RA, PA, UA and SA who attended the followup visit and healthy subjects (companions and healthcare proffesionals) were recruited. In all cases, a thermal image of the hands was taken using a Flir One Pro or Thermal Expert TE-Q1 camera connected to the mobile and an ultrasound of both hands. The degree of synovial hypertrophy (SH) and power 